Chirurg 2015 · 86:1068

DOI 10.1007/s00104-015-0095-8

Online publiziert: 13. Oktober 2015

(c) Springer-Verlag Berlin Heidelberg 2015

CrossMark

O. Strobel · M.W. Büchler

Klinik für Allgemein-, Viszeral- und Transplantationschirurgie, Universität Heidelberg,

Heidelberg, Deutschland

\title{
Peritonealer Patch als autologer Venenersatz in der Pankreas- und hepatobiliären Chirurgie
}

Schicht. Die PP-Grafts wurden als Patch oder nach Bildung eines Tubes mit der peritonealen Seite in Richtung Gefäßlumen in fortlaufender Technik mit 5/0 oder $6 / 0$ monofilem Material eingenäht. Vor Freigabe erfolgten die lokale Spülung mit Heparin und ein Flushen der Vene. Postoperativ erfolgte die prophylaktische Antikoagulation mit niedermolekularem Heparin (0,4-0,6 ml) für 4 Wochen. Neben der Erfassung perioperativer Parameter wurde insbesondere die Offenheitsrate der rekonstruierten Venen mittels Computertomographie (CT) an den postoperativen Tagen $8,30,90$ und 120 analysiert.

\section{Ergebnisse}

Es wurden 30 Patienten in die Studie eingeschlossen, davon hatten 18 komplexe Pankreasresektionen, 11 komplexe Leberresektionen und ein Patient eine kombinierte Resektion. Das PP wurde in 17 Fällen aus dem Hypochondrium und 9-mal vom Zwerchfell entnommen. In 4 Fällen wurde das Ligamentum falciforme benutzt. Von den 19 Pankreaseingriffen wurden bei 11 Pankreatoduodenektomien und 7 Pankreaslinksresektionen mit Resektion der Pfortader/V. mesenterica superior zur venösen Rekonstruktion 15 laterale Patchplastiken, 2 mehr als semizirkuläre Venenersätze und ein tubuläres Interponat aus PP verwendet. Von den 12 Major-Leberresektionen mit venöser Rekonstruktion wurden 6-mal die Pfortader, 3-mal eine Lebervene und 3-mal die V. cava rekonstruiert unter Verwendung einer Patchplastik ( $n=11$ ) oder eines tubulären Interponats aus $\mathrm{PP}(n=1)$. Es gab keine Mortalität oder Reoperation. Die Morbidität betrug $47 \%$ ( $n=14$ Patienten mit Komplikationen). Es gab eine Nachblutung, jedoch nicht aus einer rekonstruierten Ve- ne. In der letzten CT-Evaluation nach median 14 Monaten betrug die Offenheitsrate $97 \%(29 / 30)$. In $90 \%$ (27/30) zeigte sich keine oder nur eine leichte Stenose $(<25 \%)$ der rekonstruierten Vene, in 7\% $(n=2)$ eine Stenose zwischen $25-75 \%$. Ein tubuläres Interponat war verschlossen, wahrscheinlich sekundär nach Kompression durch ein Biliom. Bei 25 (86\%) von 29 Patienten mit malignen Tumoren konnte eine R0-Resektion erzielt werden.

\section{Diskussion}

Die Autoren folgern, dass die Verwendung des parietalen Peritoneums in der Leber- und Pankreaschirurgie eine vergleichsweise wenig aufwendige, rasch verfügbare, sichere und effektive Möglichkeit zur venösen Gefäßrekonstruktion darstellt, die sich insbesondere für laterale Patchplastiken anbietet.

Dieses innovative Verfahren zur venösen Rekonstruktion stellt eine wichtige Erweiterung der verfügbaren Techniken in der modernen onkologischen Pankreas- und hepatobiliären Chirurgie dar. Darüber hinaus sollte diese Technik auch in der Gefäß- und Thoraxchirurgie evaluiert werden.

\section{Korrespondenzadresse}

PD Dr. O. Strobel

Klinik für Allgemein-, Viszeral- und Transplantationschirurgie

Universität Heidelberg

Im Neuenheimer Feld 110, 69120 Heidelberg

Oliver.Strobel@med.uni-heidelberg.de

Interessenkonflikt. O. Strobel und M.W. Büchler geben an, dass kein Interessenkonflikt besteht. 